\title{
Inferring the choreography of parental genomes during fertilization from ultralarge-scale whole-transcriptome analysis
}

\author{
Sung-Joon Park, ${ }^{1}$ Makiko Komata, ${ }^{2}$ Fukashi Inoue, ${ }^{3}$ Kaori Yamada, ${ }^{3}$ Kenta Nakai, ${ }^{1}$ Miho Ohsugi, ${ }^{3,4}$ \\ and Katsuhiko Shirahige ${ }^{2,4}$ \\ ${ }^{1}$ Human Genome Center, the Institute of Medical Science, the University of Tokyo, Tokyo 108-8639, Japan; ${ }^{2}$ Laboratory of \\ Genome Structure and Function, Institute of Molecular and Cellular Biosciences, the University of Tokyo, Tokyo 113-0032, \\ Japan; ${ }^{3}$ Division of Oncology, the Institute of Medical Science, the University of Tokyo, Tokyo 108-8639, Japan
}

Fertilization precisely choreographs parental genomes by using gamete-derived cellular factors and activating genome regulatory programs. However, the mechanism remains elusive owing to the technical difficulties of preparing large numbers of high-quality preimplantation cells. Here, we collected $>14 \times 10^{4}$ high-quality mouse metaphase II oocytes and used these to establish detailed transcriptional profiles for four early embryo stages and parthenogenetic development. By combining these profiles with other public resources, we found evidence that gene silencing appeared to be mediated in part by noncoding RNAs and that this was a prerequisite for postfertilization development. Notably, we identified 817 genes that were differentially expressed in embryos after fertilization compared with parthenotes. The regulation of these genes was distinctly different from those expressed in parthenotes, suggesting functional specialization of particular transcription factors prior to first cell cleavage. We identified five transcription factors that were potentially necessary for developmental progression: Foxd1, Nkx2-5, Sox18, Myod1, and Runx1. Our very large-scale whole-transcriptome profile of early mouse embryos yielded a novel and valuable resource for studies in developmental biology and stem cell research. The database is available at http://dbtmee.hgc.jp.

[Keywords: development; fertilization; parthenogenesis; transcriptome; transcription factor]

Supplemental material is available for this article.

Received August 5, 2013; revised version accepted November 18, 2013.

Fertilization of an oocyte by a sperm is the first developmental step to confer totipotency on the newly formed embryo. Fertilization stimulates a precisely controlled coordination of maternal and paternal genomes through a sequence of different biological processes. Deciphering the mechanisms underlying this choreography of the parental genomes in mammalian embryos has long been recognized as one of the fundamental challenges in human infertility and stem cell biology research.

\footnotetext{
${ }^{4}$ Corresponding authors

E-mail kshirahi@iam.u-tokyo.ac.jp

E-mail mohsugi@bio.c.u-tokyo.ac.jp

Article is online at http://www.genesdev.org/cgi/doi/10.1101/gad.227926.113. Freely available online through the Genes \& Development Open Access option.
}

Recent technological advances in cell biology have fostered a number of studies to obtain new insights into the mechanisms in various species (Latham et al. 1991; Schultz 2002; Krawetz 2005; Wang and Dey 2006). One remarkable finding was the identification of programmed "waves" of gene expression during development. This phenomenon has been confirmed through global gene expression profiles determined with expressed sequence tag sequencing (Ko et al. 2000), microarrays (Hamatani et al. 2004; Wang et al. 2004; Zeng et al. 2004; Vassena et al. 2011), and high-throughput RNA sequencing (RNAseq) (Tang et al. 2009; Macfarlan et al. 2012; Tan et al.

(C) 2013 Park et al. This article, published in Genes \& Development, is available under a Creative Commons License (Attribution-NonCommercial 3.0 Unported), as described at http://creativecommons.org/licenses/by$\mathrm{nc} / 3.0 /$. 
2013). In mice, maternal RNAs are degraded (Piko and Clegg 1982), and then the embryos exhibit waves of gene expression transitions; namely, zygotic gene activation (ZGA) at the two-cell stage (2C) and mid-preimplantation gene activation (MGA) at the four-cell stage (4C) (Wang and Dey 2006). These gene expression patterns are altered in parthenogenetic embryos (Bui et al. 2011), leading to abortive development owing to abnormalities in genetic imprints and signal transduction (Williams et al. 1996; Fan and Sun 2004; Wang et al. 2004; Liu et al. 2010). Recent studies have also shown that parental epigenomic effects also play an essential role in establishing the appropriate expression profile during development; among these epigenetic modifications are DNA methylation (Kobayashi et al. 2012; Smith et al. 2012), histone modification (Miller et al. 2010; Santenard et al. 2010; Erkek et al. 2013), and regulation by noncoding RNAs (ncRNAs) (Amanai et al. 2006; Tang et al. 2007; Svoboda and Flemr 2010).

Although there is increasing information on the importance of the control and interaction of maternal and paternal genomes in normal development, our understanding of genomic events at very early stages of development is far from complete owing to the technical difficulties of preparing large numbers of high-quality preimplantation cells. To date, for example, relatively large-scale studies have used several hundred (Hamatani et al. 2004; Zeng et al. 2004) to a thousand (Ko et al. 2000; Kobayashi et al. 2012) embryos per stage. In addition, the embryos in these studies were not consistent with respect to their developmental time frame or cell cycle stage, bringing into question the validity of the conclusions regarding the identified molecular factors. In the present study, we sought to overcome these possible limitations by preparing very large numbers of highquality preimplantation cells in order to enable extensive genome-wide profiling with greater reliability and reproducibility.

We collected $>14 \times 10^{4}$ mouse metaphase II (MII) oocytes and, after in vitro fertilization or induced parthenogenesis, used nocodazole to precisely synchronize the cell cycle to achieve a precise analysis of specific embryonic stages. High-throughput sequencing technology was used to establish transcriptional profiles in this unprecedentedly ultralarge-scale experiment. As a result, we were able to build a database through bioinformatic analyses of this very high-quality resource. Our results confirmed that the genome activation program commenced immediately after fertilization and that this program was essential for successful development. Furthermore, our bioinformatic analyses suggested that this activation program involves maternal and sperm-borne factors as well as mRNAs newly synthesized after fertilization, all of which comprise a distinctive gene regulatory network compared with that of parthenotes. Our database will help researchers explore the genetic and epigenetic characteristics of genes involved in genome activation programs and, in particular, identify potential key transcription factors (TFs) involved in transcriptional initiation in fertilized oocytes.

\section{Results}

Whole-transcriptome analysis

Large-scale temporal RNA-seq To investigate transcriptional profiles of mouse embryos at early developmental stages, we extracted high-quality $\mathrm{MII}$ oocytes and one-cell stage (1C), 2C, and 4C embryos. In addition, we prepared parthenogenetic $1 \mathrm{C}(\mathrm{p} 1 \mathrm{C})$ and $\mathrm{p} 4 \mathrm{C}$ embryos and also used mouse embryonic fibroblasts (MEFs) (Fig. 1A). Next, we performed SOLiD RNA-seq with ribosomal RNA (rRNA)depleted total RNA libraries. This RNA-seq of mouse embryos was performed on an extremely large scale using 10,000 cells per replicate, with two or three replicates per stage.

From this large-scale analysis, we generated 139.9 million-229.8 million 50-base-pair (bp) reads (tags) from the replicates of each developmental stage (Table 1). Approximately $62.9 \%-67.6 \%$ of the sequenced reads were mapped to the mouse genome (University of California at Santa Cruz [UCSC] $\mathrm{mm}$ 9) by TopHat (Trapnell et al. 2012); we increased the fraction of uniquely mapped reads (unireads) by $8.8 \%-15.1 \%$ using a recursive mapping strategy. These unireads were used for further analysis. Compared with previous studies (Tang et al. 2009; Macfarlan et al. 2012), our RNA-seq data gave higher coverage and/or depth (Supplemental Table S1); 43.9\%$48.4 \%$ of the 59.3 million nonredundant RefSeq exonic bases were covered by at least one uniread, and, on average, a given exonic base position had more than three unireads.

Characterization of the transcriptional profile We used the Cufflinks package (Trapnell et al. 2012) to quantify RefSeq transcripts; this analysis confirmed high reproducibility among the replicates (Supplemental Table S2) and obtained a compendium of the transcriptional profile during development (Supplemental Fig. S1). To validate the RNA-seq results, we performed quantitative RT-PCR (qRT-PCR) on 16 randomly selected genes and found that 11 genes were in good agreement (Supplemental Fig. S2). In addition, we found striking concordance of expression patterns with well-known genes that have been validated previously (Supplemental Fig. S2). Of note, the comparison with qRT-PCR shows variance in particular at $2 \mathrm{C}$, presumably due to the fact that a large fraction of the maternally supplied mRNAs has been degraded at this stage (Piko and Clegg 1982).

As a characteristic of the transcriptional profile, a dramatic change in expression occurred during the transition from 1C to 2C (Supplemental Fig. S1), which is consistent with descriptions from previous studies (Latham et al. 1991; Schultz 2002). Additionally, the total number of bases aligned by unireads decreased at $2 \mathrm{C}$ but subsequently increased at $4 \mathrm{C}$ (Fig. 1B); this pattern of change is consistent with the variation in the amount of RNA during development (Piko and Clegg 1982; Tang et al. 2007; Svoboda and Flemr 2010). Interestingly, a peculiar distribution of unireads, in which intronic and intergenic reads were sequentially increased, was identified (Fig. 1B); this is consistent with the results of a recent mRNA-seq assay (Supplemental Fig. S3). We suggest that oocytes and 
Park et al.
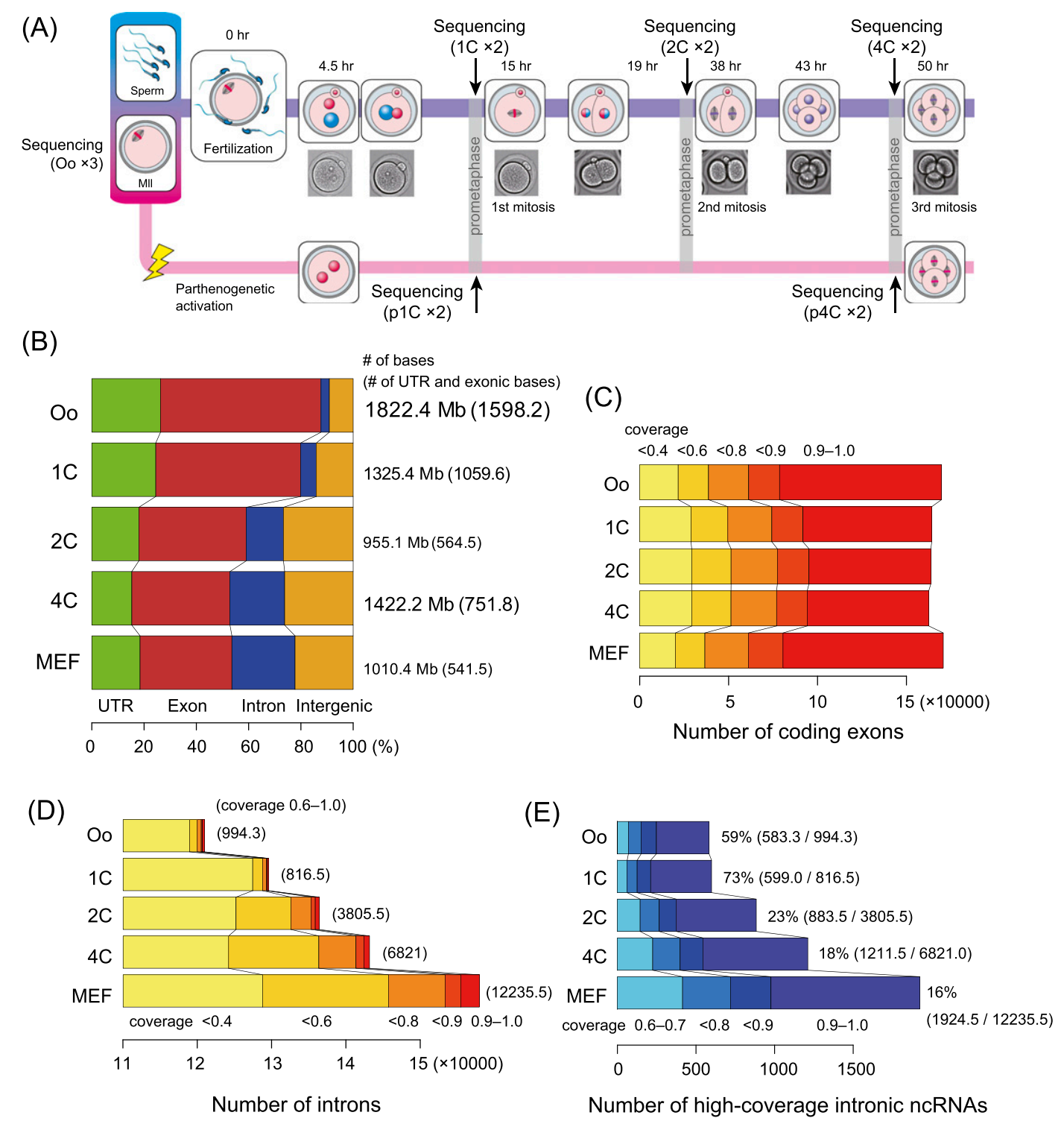

Figure 1. Systematic representation of transcriptome analysis and summary of mapped read distributions. $(A)$ Developmental stages targeted by the RNA-seq assay. $(B)$ Distribution of uniquely mapped reads along mouse genomic features. $(C, D)$ Histograms showing the number of RefSeq exons and introns and the coverage levels. (E) Histogram showing the number of intronic ncRNAs (coverage $>0.6)$ and their proportion among the total detected introns shown in $D$. Averaged numbers across replicates are shown. (Oo) Oocyte.

1C mainly contain fully matured maternal mRNAs, while the rise in intronic reads at $2 \mathrm{C}$ and $4 \mathrm{C}$ may reflect an increasing level of nascent transcription. More specifically, the number of exons aligned by the reads was relatively consistent at different stages (Fig. 1C), whereas more introns were detected with higher coverage as the embryos developed (Fig. 1D). Interestingly, oocytes and 1C exhibited a higher percentage of ncRNAs than later stages (Fig. 1E). This may suggest that ncRNAs have a distinct and important role during development.

Extensive transcriptome discovery To address the nonexonic reads illustrated in Figure 1B, we assembled and quantified ncRNAs using comprehensive annotation (Kin et al. 2007). We found that many ncRNAs were tran- scribed at high levels during development (Fig. 2A). The vast majority were miscellaneous RNAs (miscRNAs) or antisense RNAs (asRNAs) (Table 2). A dendrogram analysis of the expression profiles of the ncRNAs revealed a major transition in expression between $1 \mathrm{C}$ and $2 \mathrm{C}$ (Fig. $2 \mathrm{~B})$; the analysis also indicated that fertilization might influence this major transition because 1C was located far from the cluster of oocytes and p1C. This putative fertilization effect appeared to be time-limited, as 4C and $\mathrm{p} 4 \mathrm{C}$ were clustered in all cases.

Mature microRNAs (miRNAs) were too short to be sequenced here. Indeed, the maternal miRNA let-7 family (Tang et al. 2007) was not detected. However, several miRNA precursors were identified (Table 2); in particular, mir-290 (Svoboda and Flemr 2010) appeared at 4C and 
Table 1. Summary of sequencing data

\begin{tabular}{|c|c|c|c|c|c|c|c|c|c|}
\hline \multirow[b]{2}{*}{ Stage-replicate ID } & \multirow[b]{2}{*}{ Raw reads } & \multicolumn{4}{|c|}{ Mapping (50 bp) } & \multicolumn{2}{|c|}{ Recursive mapping } & \multicolumn{2}{|c|}{ Improvement } \\
\hline & & Mapped & Percentage & Uniread & Percentage & Uniread & Percentage & Uniread & Percentage \\
\hline Oocyte-1 & $79,873,808$ & $49,347,945$ & $61.8 \%$ & $35,115,124$ & $44.0 \%$ & $48,340,264$ & $60.5 \%$ & $13,225,140$ & $16.6 \%$ \\
\hline Oocyte-2 & $96,416,177$ & $61,076,888$ & $63.4 \%$ & $41,804,743$ & $43.4 \%$ & $56,386,539$ & $58.5 \%$ & $14,581,796$ & $15.1 \%$ \\
\hline Oocyte-3 & $53,514,580$ & $36,436,197$ & $68.1 \%$ & $25,833,980$ & $48.3 \%$ & $32,812,131$ & $61.3 \%$ & $6,978,151$ & $13.0 \%$ \\
\hline Oocyte (total) & $229,804,565$ & $146,861,030$ & $63.9 \%$ & $102,753,847$ & $44.7 \%$ & $137,538,934$ & $59.9 \%$ & $34,785,087$ & $15.1 \%$ \\
\hline $1 \mathrm{C}-1$ & $101,479,447$ & $60,478,506$ & $59.6 \%$ & $29,653,767$ & $29.2 \%$ & $41,385,327$ & $40.8 \%$ & $11,731,560$ & $11.6 \%$ \\
\hline $1 \mathrm{C}-2$ & $61,823,156$ & $42,190,486$ & $68.2 \%$ & $25,838,554$ & $41.8 \%$ & $32,486,931$ & $52.6 \%$ & $6,648,377$ & $10.8 \%$ \\
\hline 1C (total) & $163,302,603$ & $102,668,992$ & $62.9 \%$ & $55,492,321$ & $34.0 \%$ & $73,872,258$ & $45.2 \%$ & $18,379,937$ & $11.3 \%$ \\
\hline $2 \mathrm{C}-1$ & $97,579,611$ & $60,561,715$ & $62.1 \%$ & $28,392,629$ & $29.1 \%$ & $36,879,889$ & $37.8 \%$ & $8,487,260$ & $8.7 \%$ \\
\hline $2 \mathrm{C}-2$ & $42,340,542$ & $28,836,186$ & $68.1 \%$ & $15,517,129$ & $36.7 \%$ & $19,404,875$ & $45.8 \%$ & $3,887,746$ & $9.2 \%$ \\
\hline 2C (total) & $139,920,153$ & $89,397,901$ & $63.9 \%$ & $43,909,758$ & $31.4 \%$ & $56,284,764$ & $40.2 \%$ & $12,375,006$ & $8.8 \%$ \\
\hline $4 C-1$ & $111,479,302$ & $75,817,830$ & $68.0 \%$ & $40,427,154$ & $36.3 \%$ & $50,788,951$ & $45.6 \%$ & $10,361,797$ & $9.3 \%$ \\
\hline $4 C-2$ & $66,859,932$ & $44,692,078$ & $66.8 \%$ & $25,374,399$ & $38.0 \%$ & $31,776,191$ & $47.5 \%$ & $6,401,792$ & $9.6 \%$ \\
\hline 4C (total) & $178,339,234$ & $120,509,908$ & $67.6 \%$ & $65,801,553$ & $36.9 \%$ & $82,565,142$ & $46.3 \%$ & $16,763,589$ & $9.4 \%$ \\
\hline MEF-1 & $43,287,853$ & $26,389,019$ & $61.0 \%$ & $14,475,245$ & $33.4 \%$ & $19,598,458$ & $45.3 \%$ & $5,123,213$ & $11.8 \%$ \\
\hline MEF-2 & $63,906,069$ & $40,648,118$ & $63.6 \%$ & $27,900,833$ & $43.7 \%$ & $37,182,810$ & $58.2 \%$ & $9,281,977$ & $14.5 \%$ \\
\hline MEF (total) & $107,193,922$ & $67,037,137$ & $62.5 \%$ & $42,376,078$ & $39.5 \%$ & $56,781,268$ & $53.0 \%$ & $14,405,190$ & $13.4 \%$ \\
\hline p1C-1 & $49,374,159$ & $24,859,290$ & $50.4 \%$ & $12,531,604$ & $25.4 \%$ & $18,711,095$ & $37.9 \%$ & $6,179,491$ & $12.5 \%$ \\
\hline p1C-2 & $52,235,287$ & $27,552,654$ & $52.8 \%$ & $14,497,667$ & $27.8 \%$ & $20,920,391$ & $40.1 \%$ & $6,422,724$ & $12.3 \%$ \\
\hline p1C (total) & $101,609,446$ & $52,411,944$ & $51.6 \%$ & $27,029,271$ & $26.6 \%$ & $39,631,486$ & $39.0 \%$ & $12,602,215$ & $12.4 \%$ \\
\hline $\mathrm{p} 4 \mathrm{C}-1$ & $55,440,412$ & $33,837,966$ & $61.0 \%$ & $16,620,244$ & $30.0 \%$ & $21,740,003$ & $39.2 \%$ & $5,119,759$ & $9.2 \%$ \\
\hline $\mathrm{p} 4 \mathrm{C}-2$ & $50,882,120$ & $32,726,783$ & $64.3 \%$ & $16,711,193$ & $32.8 \%$ & $21,067,528$ & $41.4 \%$ & $4,356,335$ & $8.6 \%$ \\
\hline $\mathrm{p} 4 \mathrm{C}$ (total) & $106,322,532$ & $66,564,749$ & $62.6 \%$ & $33,331,437$ & $31.3 \%$ & $42,807,531$ & $40.3 \%$ & $9,476,094$ & $8.9 \%$ \\
\hline
\end{tabular}

p4C, suggesting a fertilization-independent function. The mir-17 family was specifically transcribed at 4C but not $\mathrm{p} 4 \mathrm{C}$, suggesting that this is a candidate zygotic miRNA initiated by fertilization. Additionally, stage-specific ncRNAs were identified more frequently than coding RNAs in fertilized oocytes (Fig. 2C).
Next, we sought to assess the overlap of our RNA-seq assay with public array-based profiles (Hamatani et al. 2004; Zeng et al. 2004). As shown in Figure 2D, our RNAseq assay covered almost all of the genes detected with microarrays. Our assay uniquely identified 5364 genes that were expressed at oocytes, 1C, or 2C. Here, we detected
(A) Total number of transcripts

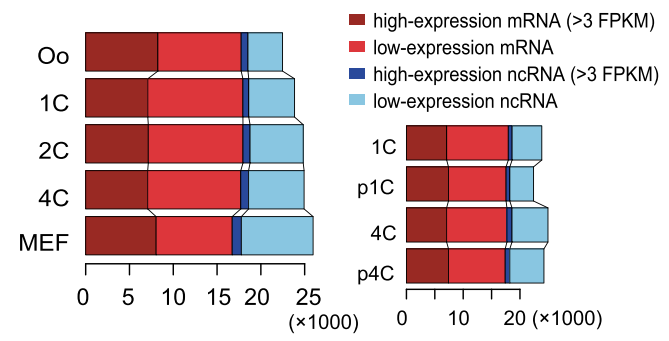

(B) Stage clustering based on ncRNA expressions

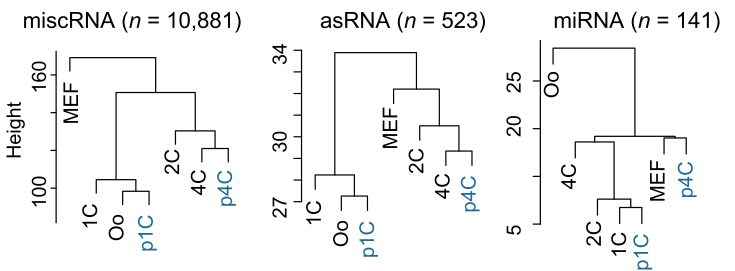

(C) Number of stage-specific transcripts

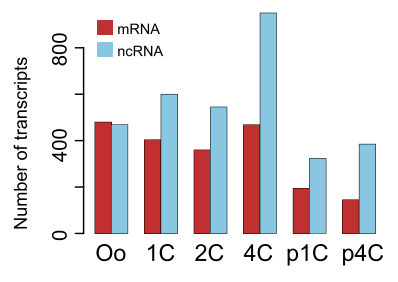

(D) Comparision of RNA-seq to microarray

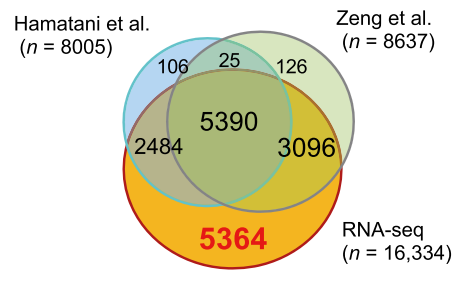

Figure 2. Summary of transcriptome discovery. $(A)$ The histogram shows the total number of mRNAs and ncRNAs discovered in the present study. (B) Cluster analysis for the major ncRNAs shows dramatic expression changes between 1C and 2C, except for miRNAs, whose small size precluded analysis. $(C)$ ncRNAs are more actively transcribed than mRNAs. Relatively few RNAs are specifically transcribed in p1C and p4C embryos compared with normal development. (D) Our RNA-seq assay discovered 5364 mRNAs that are not detected with microarrays. (FPKM) Fragments per kilobase of exon per million mapped reads, a unit of digital gene expression level; (Oo) oocyte. 
Park et al.

Table 2. Major category of ncRNA species profiled with the RNA-seq assay

\begin{tabular}{lrrrrrrr}
\hline Category & Oocyte & \multicolumn{1}{c}{$1 \mathrm{C}$} & \multicolumn{1}{c}{ 2C } & \multicolumn{1}{c}{ 4C } & MEF & p1C & p4C \\
\hline miscRNA & 4128 & 5242 & 6184 & 6547 & 8428 & 4259 & 6196 \\
asRNA & 236 & 273 & 269 & 245 & 310 & 233 & 215 \\
snoRNA & 152 & 148 & 159 & 167 & 164 & 153 & 160 \\
Pseudo & 142 & 143 & 140 & 135 & 108 & 127 & 134 \\
miRNA & 18 & 19 & 40 & 46 & 112 & 15 & 46 \\
snRNA & 10 & 10 & 10 & 12 & 11 & 9 & 8 \\
Total & 4686 & 5835 & 6802 & 7152 & 9133 & 4796 & 6759 \\
\hline
\end{tabular}

4125 genes at oocytes, whereas other profiles detected a much smaller set of genes at this stage: $36.3 \%$ (1496) with an Agilent microarray platform (Pfeiffer et al. 2011), $39.7 \%$ (1636) with a single-cell SOLiD system (Tang et al. 2009), and 31.9\% (1317) with an Illumina GA (Macfarlan et al. 2012). This comparison indicated that the present study successfully identified a more complete picture of the transcriptome than was achieved by previous studies.

\section{Analysis of differential gene expression}

Pairwise comparisons suggest repressive and selective expression changes To analyze differentially expressed genes, we applied Cuffdiff (Trapnell et al. 2012) to our RNA-seq data set (Fig. 3A). Using a threshold of 1.5-fold change (Liu et al. 2010), the analysis showed a transcriptionally repressive state: 3798 maternal transcripts were down-regulated in the oocyte-to-1C transition, reflecting rapid degradation of maternal RNAs. Additionally, 5950 maternal transcripts were down-regulated throughout $2 \mathrm{C}$ and $4 \mathrm{C}$, and 4967 and 3541 transcripts were repressed during the $1 \mathrm{C}$-to-2C and $2 \mathrm{C}$-to-4C transitions, respectively. The numbers of transcripts showing repression were markedly higher than those showing up-regulation during the transitions, even when more stringent thresholds were used.

Our analysis showed that certain transcripts appeared immediately after fertilization. A few transcripts (596) were up-regulated during the oocyte-to-1C transition; however, $64 \%$ (383 out of 596) of these were not upregulated during the oocyte-to-p1C transition. Since most of the transcripts (380 out of 383) exhibited a $>1.5$-fold suppression following treatment with the transcription inhibitor $\alpha$-amanitin (Supplemental Fig. S7B), we speculated that the up-regulation reflected nascent transcription by minor ZGA (Wang and Dey 2006). However, 329 out of the 383 transcripts are also expressed in sperm (Kobayashi et al. 2012). Since these transcripts did not appear in p1C embryos, we conclude that coordination between minor ZGA and sperm-borne RNAs contributed to the increased RNAs at $1 \mathrm{C}$ and suggest that the upregulated transcripts at $1 \mathrm{C}$ were "primed" by the sperm. Approximately $45 \%$ (173 out of 383 ) of the transcripts also showed up-regulation during the 1C-to-2C transition; as 150 of these were expressed in sperm, this again suggested that the majority were sperm-primed nascent transcripts. These genes included Spz1, Smad7, and the
(A)

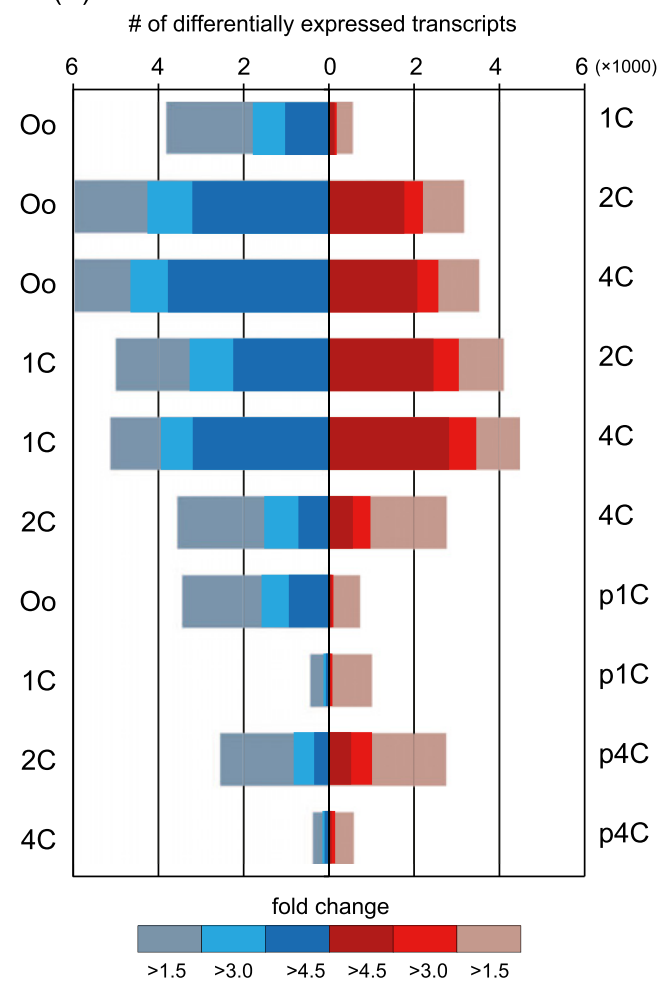

(B) Down-regulated at p1C vs. 1C

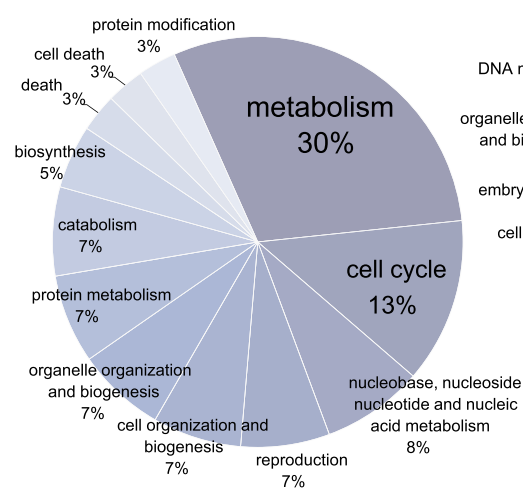

(D) Down-regulated at $\mathrm{p} 4 \mathrm{C}$ vs. $4 \mathrm{C}$

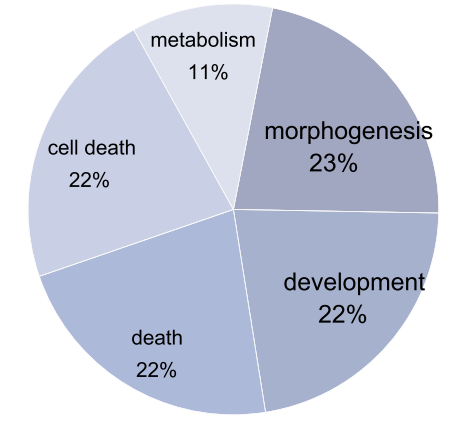

(C) Up-regulated at p1C vs. $1 \mathrm{C}$

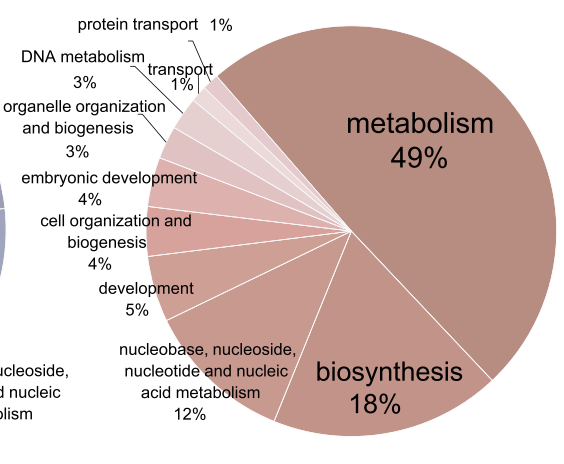

(E) Up-regulated at p4C vs. $4 \mathrm{C}$

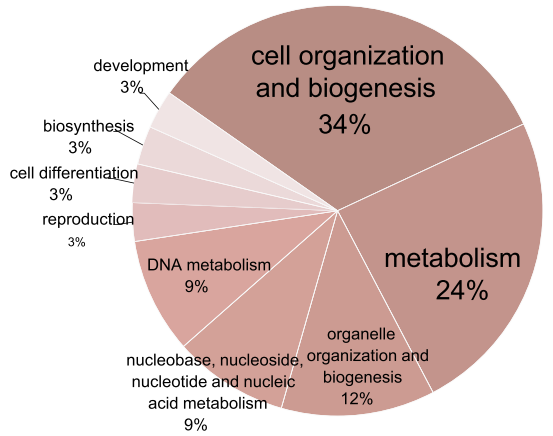

Figure 3. Gene expression changes and significant parthenogenetic biological processes. $(A)$ Differential gene expression in all of the possible pairs. $(B-E)$ Representative GO:BP terms enriched in parthenotes (fold change > 1.5). (Oo) Oocyte. 
potential pluripotency regulators Pramel1, Pramel6, and Pramel7 (Wang et al. 2001; Casanova et al. 2011; Hirata et al. 2012).

We also identified a dramatic change in expression during the 1C-to-2C transition, in which 4967 and 4082 transcripts were down-regulated and up-regulated, respectively, at $2 \mathrm{C}$ relative to $1 \mathrm{C}$. The three genes showing the greatest down-regulation at 2C were Psrc1, Dnmt3a, and Acs13, and the most highly up-regulated genes at this stage were Dppa4, Timd2, and Rp134-ps1. This substantial change is possibly coupled to the onset of the major ZGA that replaces maternal RNAs with zygotic RNAs (Latham et al. 1991; Schultz 2002).

Parthenotes show a distinct pattern of gene expression A pairwise comparison of $1 \mathrm{C}$ and p1C (Fig. 3A) identified 2.3 times more up-regulated transcripts than down-regulated transcripts at $\mathrm{p} 1 \mathrm{C}$ relative to $1 \mathrm{C}$; however, this difference was reduced to 1.5 times in a 4C-to-p4C comparison. We analyzed the overrepresented gene ontology biological process (GO:BP) terms among these differentially expressed genes using DAVID (Database for Annotation, Visualization, and Integrated Discovery) (Huang et al. 2009) and categorized them into representative GO terms using CateGOrizer (Hu et al. 2008). This analysis showed that at p1C, parthenogenesis repressed expression of genes involved in the cell cycle, apoptosis, and protein metabolism (Fig. 3B) but activated genes involved in biosynthesis and protein transport (Fig. 3C). At p4C, the repression of apoptosis-related genes was still present (Fig. 3D), and chromatin-related processes were activated (Fig. 3E).

Our results are consistent with a previous report that parthenotes exhibit a high level of mRNA production at $2 \mathrm{C}$ and delay degradation to the normal level until late in 4C (Bui et al. 2011). Furthermore, our GO term analysis revealed that parthenotes must restrain zygotic cell division and preferentially recruit maternal proteins and mRNAs. Therefore, successful zygotic progression is likely under the control of aberrant gene expression as well as appropriate recruitment of pre-existing maternal and other factors.

\section{Clustering of global gene expression patterns}

The zygotic transcription program consists of alternating waves To assess global gene expression patterns in the embryos, we performed a hierarchical clustering analysis of the normalized RNA abundance of RefSeq genes (Fig. 4A); this analysis identified 25 clusters (Supplemental Fig. S4). We categorized the clusters using the categories of programmed waves suggested by Wang and Dey (2006); namely, maternal RNA (clusters 1-4), minor ZGA (clusters 5-8), major ZGA (clusters 14-16), MGA (clusters 21 and 22), stage-specific transients (clusters 2325 ), and the transitional patterns minor ZGA to MGA (clusters 9-11), maternal to ZGA (clusters 12 and 13), and maternal to MGA (clusters 17-20).

Our analysis revealed gene expression patterns that had not been defined in previous studies (Fig. 4B). For example, $94.3 \%$ of the maternal RNAs were rapidly degraded at $2 \mathrm{C}$ (clusters 1 and 2); degradation of the remainder was delayed and completed at 4C (clusters 3 and 4). The major ZGA pattern showed peak RNA levels at 2C following either steady (cluster 14) or dramatic (clusters 15 and 16) increases; these levels then declined. The MGA pattern included a rapid increase from $2 \mathrm{C}$ (cluster 21) and a steady increase by $4 \mathrm{C}$ (cluster 22). In the minor ZGA pattern, cluster 5 showed a rapid decline in RNAs at $2 \mathrm{C}$ compared with clusters $6-8$. It is possible that genes in cluster 5 undergo substantial translation, which is essential for minor ZGA (Hamatani et al. 2004). Some genes in the minor ZGA pattern were actively transcribed again at 4C (cluster 10) to contribute to MGA (minor ZGA to MGA).

The minor ZGA wave initiates a range of developmental processes To search for correlations between expression patterns and developmental processes, we first prepared gene subsets that were highly correlated with the cluster means (Pearson's correlation $>0.95$ ). Next, we performed a GO term enrichment analysis. This analysis yielded 36 GO terms representing 202 GO:BP terms found from DAVID (Fig. 4C; Supplemental Table S3).

An overview of the general and specific roles of the expression waves in development is presented in Figure 4C. Basal processes (biosynthesis and metabolism) were prevalent in the patterns. Cell communication processes that involve signal transduction were required for not only oocyte maturation (Zeng et al. 2004; Gilchrist 2011) but also zygote developmental competence (Fig. 4C, [1]). Stage-specific transients showed a response to stress and external stimuli (Fig. 4C, [2]). In addition, transitional maternal mRNAs were involved in membrane-related processes as well as the production of pyruvate, lactate, and triglycerides (Fig. 4C, [3]). The energy supply pathway is directly relevant to development (Sturmey et al. 2009; Ufer and Wang 2011).

Remarkably, the minor ZGA pattern was involved in a wide range of biological processes that are initiated upon fertilization (Fig. 4C, [4]). Some of the processes were enriched again during the minor ZGA-to-MGA transition, indicating the possible functional importance of reactivated minor ZGA genes. Thus, genes enhanced at $1 \mathrm{C}$ may have an essential impact on not only the onset of gene activation but also the control of zygotic progression.

\section{Discovering fertilization-specific mRNAs and their functions}

Categorizing transcripts first detected after oocyte activation To examine the potential link between fertilization and transcriptional initiation, we searched for genes that were not expressed at oocytes but were expressed at $1 \mathrm{C}$ and/or in sperm. We used public mRNA-seq data for spermatozoa (Kobayashi et al. 2012) and then profiled the expression levels of transcripts at oocytes, 1C, and p1C and in spermatozoa. We then retrieved the transcripts that indicated that RNAs and their proteins were not detected at oocytes in our profile and public proteome data (Wang et al. 2010; Pfeiffer et al. 2011). Next, we categorized the 
Park et al.

(A)

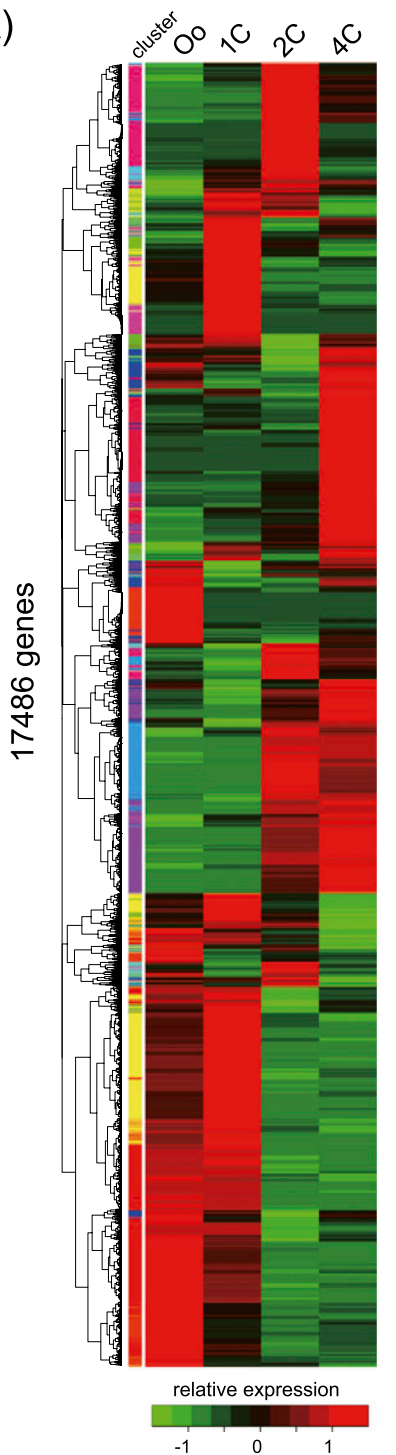

(B)

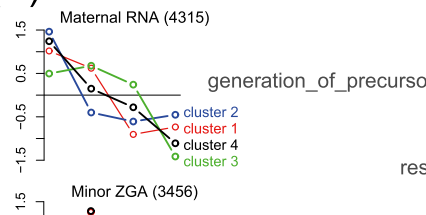

(C)
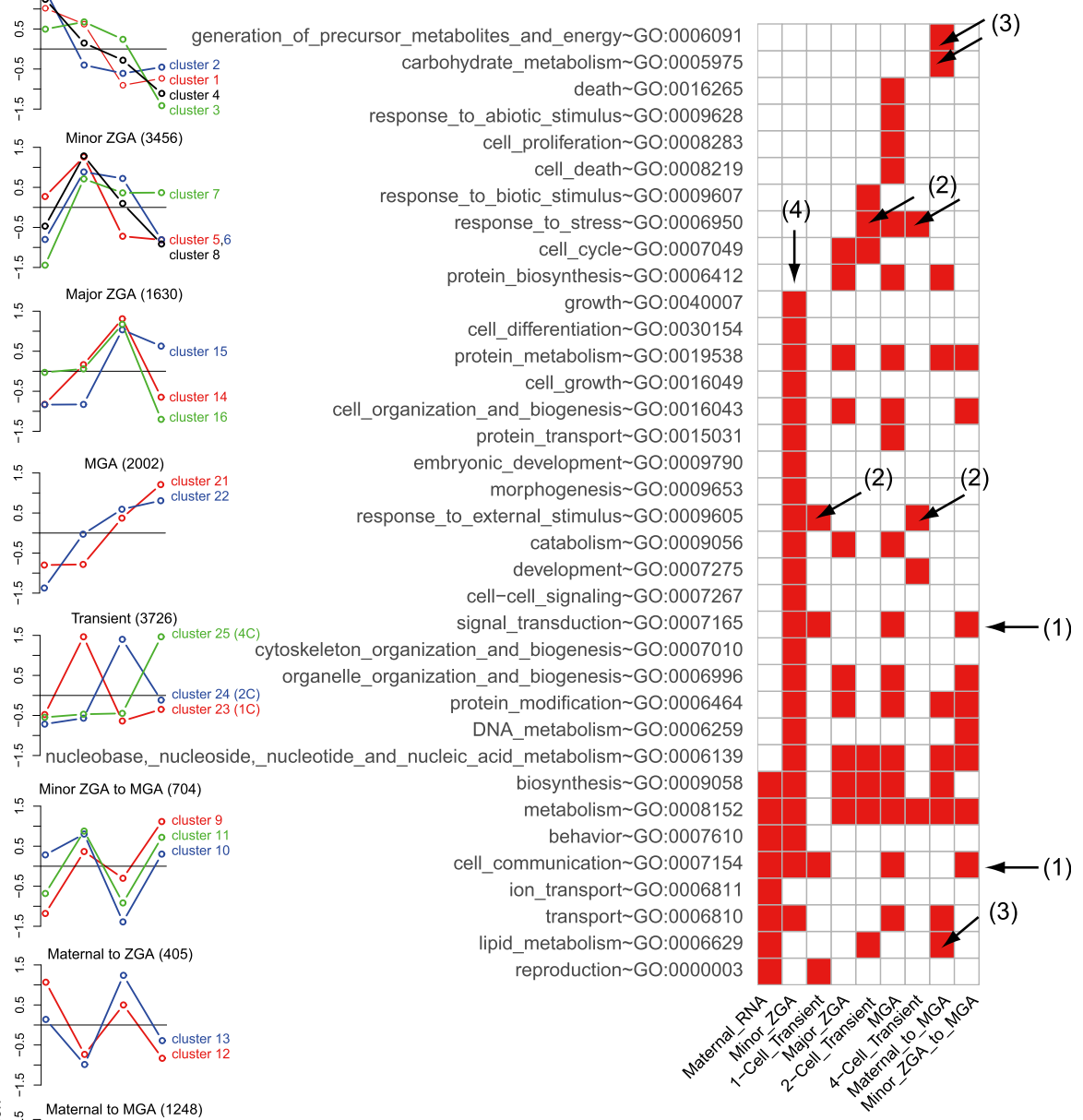

i

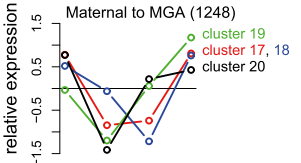

Oo $1 \mathrm{C} 2 \mathrm{C} \quad 4 \mathrm{C}$

Figure 4. Temporal gene expression patterns and their functional associations. $(A, B)$ Hierarchical clustering of normalized expression levels results in 25 expression patterns. $(C)$ Representative GO terms enriched in each pattern $(P<0.01)$ showing that minor ZGA is involved in broad developmental processes. (Oo) Oocyte.

transcripts into six groups according to their expression states, as described in Table 3 (Nsc: nascent, representing mRNAs that appeared after oocyte activation; Spr: sperm, representing mRNAs present in spermatozoa). To clarify the involvement of these transcripts during development, we clustered their expression changes into four waves (Table 3; Supplemental Table S4): 1C transient (cluster I), activation increasing to $4 \mathrm{C}$ (cluster II), $2 \mathrm{C}$ transient (cluster III), and transition fluctuating from 1C to 4C (cluster IV).

Functional analysis reveals sperm-delivered signals We analyzed GO term enrichment for the categorized genes and found 26 terms representing 144 GO:BP terms from DAVID (Supplemental Table S5). Three principal points emerged from the results. First, genes expressed at p1C are involved in a wide range of biological processes, many of which were also found at $1 \mathrm{C}$. These include male-specific reproduction processes, even though a paternal genome was not present (e.g., Acsbg2, Adam18, Adam24, Spata20, Stra8, and Taf7l in Spr1C-NscP1C). Second, genes in Nsc1C were involved in phospholipase activity and G-proteincoupled receptor (GPCR) signaling pathways known to trigger $\mathrm{Ca}^{2+}$ oscillations (Williams et al. 1996; Saunders et al. 2002). By mapping the categorized genes onto the calcium signaling pathway in KEGG (Kyoto Encyclopedia of Genes and Genomes) (Kanehisa et al. 2012), we confirmed that the gene usage of fertilized oocytes differed from that of parthenotes for GPCR in the pathway (Supplemental Fig. S5A). Unexpectedly, Plcz1 (Ramadan et al. 2012) was detected in the map as in Spr1C-NscP1C, suggesting that parthenotes also use this putative spermspecific phospholipase. Finally, genes in SprlC were highly involved in processes initiated during minor ZGA. Mitogen-activated protein kinase (MAPK) activity was the 
Table 3. Six categories of genes first detected after oocyte activation

\begin{tabular}{|c|c|c|c|c|c|c|c|c|c|}
\hline \multirow[b]{2}{*}{ Category } & \multicolumn{3}{|c|}{ Expression } & \multicolumn{4}{|c|}{ Cluster } & \multirow{2}{*}{$\frac{\text { Total }}{\text { Trans }^{\mathrm{a}} \text { (gene) }}$} & \multirow[b]{2}{*}{ Implication } \\
\hline & $1 \mathrm{C}$ & Sperm & $\mathrm{p} 1 \mathrm{C}$ & I & II & III & IV & & \\
\hline $\mathrm{Nsc1C}^{\mathrm{b}}$ & Yes & & & 116 & 109 & 78 & 18 & $321(315)$ & Nascent (Nsc) at $1 \mathrm{C}$ \\
\hline SprlC & Yes & Yes & & 194 & 163 & 110 & 46 & $513(502)$ & Sperm (Spr)-borne \\
\hline Nsc1C-NscP1C & Yes & & Yes & 56 & 53 & 47 & 20 & $176(172)$ & Nascent at $1 \mathrm{C}$; nascent at $\mathrm{p} 1 \mathrm{C}$ \\
\hline Spr1C-NscP1C & Yes & Yes & Yes & 172 & 156 & 137 & 46 & $511(501)$ & Sperm-borne; nascent at p1C \\
\hline NscP1C & & & Yes & & & & & $138(135)$ & Nascent at p1C \\
\hline SprP1C & & Yes & Yes & & & & & $250(246)$ & Detected in sperm; nascent at p1C \\
\hline
\end{tabular}

${ }^{\mathrm{a}}$ Transcript

${ }^{\mathrm{b}} \mathrm{Nsc} X$ represents RNAs first detected at $X$ stage but not oocytes.

${ }^{\mathrm{c}} \mathrm{Spr} Y$ represents RNAs present in sperm and $Y$ stage but not oocytes.

most prominent, a finding consistent with previous reports (Sun et al. 1999; Fan and Sun 2004; Liu et al. 2010). By mapping the genes onto the KEGG pathway, we also identified three extracellular signaling molecules that were not detected at oocytes (Supplemental Fig. S5B): brain-derived neurotrophic factor (Bdnf), Fas ligand (Fasl), and Cd14 antigen. Bdnf (Kawamura et al. 2005) was in the activation pattern (cluster II), suggesting an increased requirement for Bdnf during development. In contrast, Fasl (Kelkar et al. 2003) and Cd14 were in the 1C transient (cluster I) and 2C transient (cluster III) patterns, respectively. These results suggested that some sperm-borne factors localized on the sperm cell surface are related to apoptotic and immunological pathways that are depleted during parthenogenetic development.

\section{Identification of specific promoter signatures for successful development}

Nsc1C gene promoters contain highly distinctive TFbinding motifs The promoter architecture of genes from the six categories (Table 3) were analyzed for enrichment of JASPAR TF-binding motifs (Vlieghe et al. 2006) in the core promoter sequences spanning -1000 to $+200 \mathrm{bp}$ from the transcription start sites (TSSs). These sites have been previously shown to be hypomethylated in germ cells
(Kobayashi et al. 2012). We found 103 significant motifs (Fig. 5). Interestingly, Nsc1C exhibited highly distinct motifs that were particularly prominent in the $1 \mathrm{C}$ transient (cluster I) and activation (cluster II) patterns. In addition, TBP- and Sp1-binding motifs, which are thought to be required after fertilization (Worrad et al. 1994; Gazdag et al. 2007), appeared in different gene categories: TBP-dependent genes in Nsc1C and Sp1-dependent genes in Spr1C and Spr1C-NscP1C. Parthenotes (NscP1C, SprP1C, and Spr1C-NscP1C) showed Sp1-binding motifs but not TBP-binding motifs. These results suggest that the regulatory codes for TFs during normal development are distinctively different from those in parthenogenetic development.

Five TFs potentially important for initial gene activation We searched for TFs that might be important for regulating the six gene categories by constructing a network of TFs known to bind to the JASPAR motifs in TRANSFAC (Wingender et al. 2000). The network was composed of 53,229 edges among 1897 nodes (Fig. 6A; Supplemental Fig. S6). The nodes consisted of 97 maternal TFs, 11 nonmaternal TFs first detected after oocyte activation, and their target genes. Additionally, eight of the 11 nonmaternal TFs showed self-regulation (Fig. 6C), suggesting a requirement for

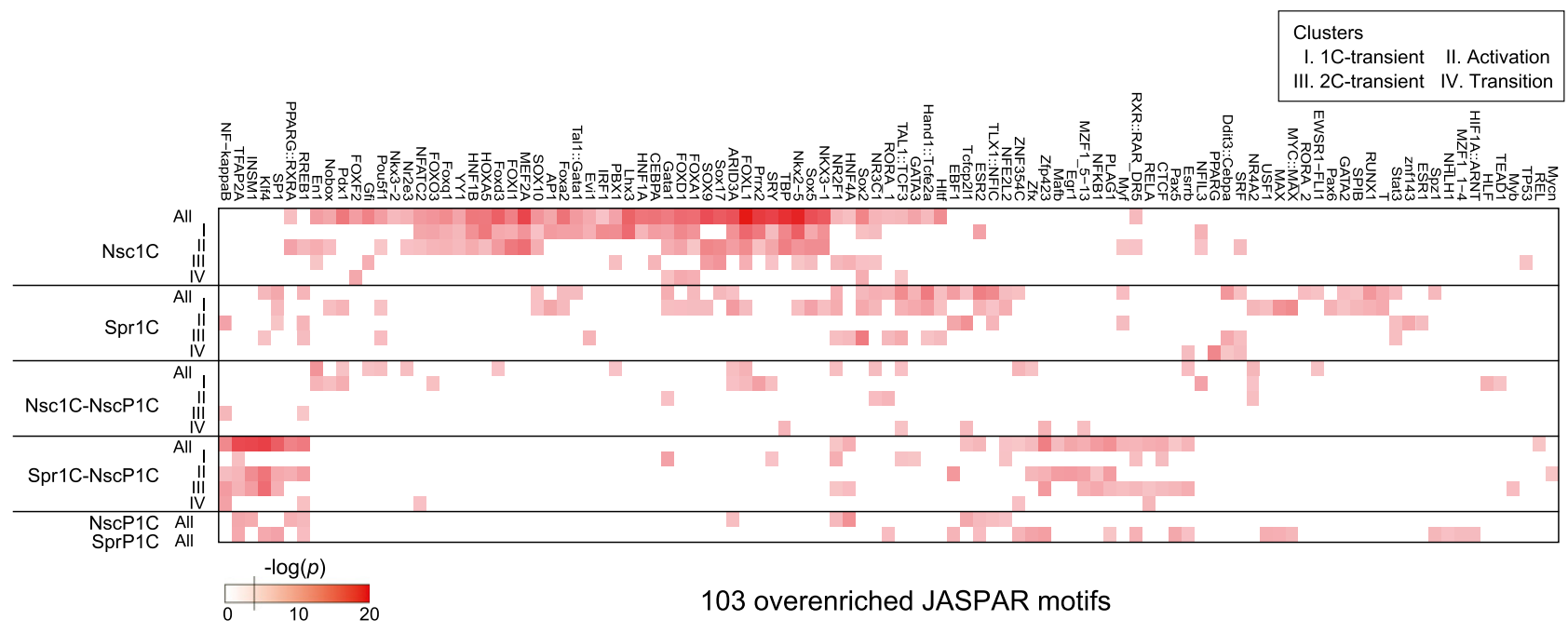

Figure 5. Analysis of TF-binding motif enrichment. Heat map showing enriched TF-binding motifs in core promoters $(P<0.01)$. (Nsc) Nascent; (Spr) sperm. 
Park et al.

a "kickoff" TF to regulate itself. Sperm may prime the self-regulation to enable rapid responses, whereas nascent and parthenogenetic TFs may be modulated by alternative mechanisms.

Higher TF-TF connectivity in the network may contribute to such alternative mechanisms. The clustering coefficient of a given gene quantifies the level of TF connectivity; i.e., the interregulation of TFs required for gene regulation. For example, as shown in Figure 6A, Pcdhgb2 contained motifs that are targeted by 11 maternal and three specific nonmaternal TFs (Mafb, Egr3, and Rreb1). The maternal TFs are suggested to simultaneously regulate the three nonmaternal TFs. Thus, expression of Pcdhgb2 can be properly modulated even if the specific TFs are initially absent in parthenotes. On average, a higher CC $(>0.1)$ was observed in SprlCNscP1C (Fig. 6B), indicating that TFs necessary for commonly expressed genes in sperm and $1 \mathrm{C}$ and $\mathrm{plC}$ embryos tightly modulate each other.

The most prevalent maternal TFs were high-mobility group (HMG) proteins (TCF and Sox families) and their cooperative partners (C/EBP and Sp/Klf families) (Fig. 6C), suggesting a significant role for nonhistone chromosomal proteins (Bianchi and Beltrame 1998; Beaujean et al. 2000; Gaillard and Strauss 2000). In contrast, Ap-1 family members specifically target Nsc1C genes and may mediate rapid stimulus response and cell development (Hess et al. 2004). The regulation of gene expression in Nsc1C particularly involved Foxd1 and Nkx2-5, which were expressed at 1C. This regulation involved Sox18 (Spr1C category) and Myod1 (Nsc1C category), which were also required for Spr1C genes. Our analysis suggested that Runx1 (Spr1C category) regulated Spr1C genes. Nfatc2, $H n f 1 a$, and Sox 9 appeared in parthenotes. Taken together, Foxd1 $(\mathrm{CC}=0.14), N k x 2-5(\mathrm{CC}=0.13)$, Sox18 $(\mathrm{CC}=$ $0.052)$, Myod1 (CC $=0.034)$, and Runx1 ( $\mathrm{CC}=0.058$ ) are suggested to be potentially key TFs for successful development. In good agreement with this suggestion, these five TFs were not observed in parthenogenesis.

\section{Discussion}

Fertilization induces embryonic developmental competence in oocytes through precise and sequential regu-

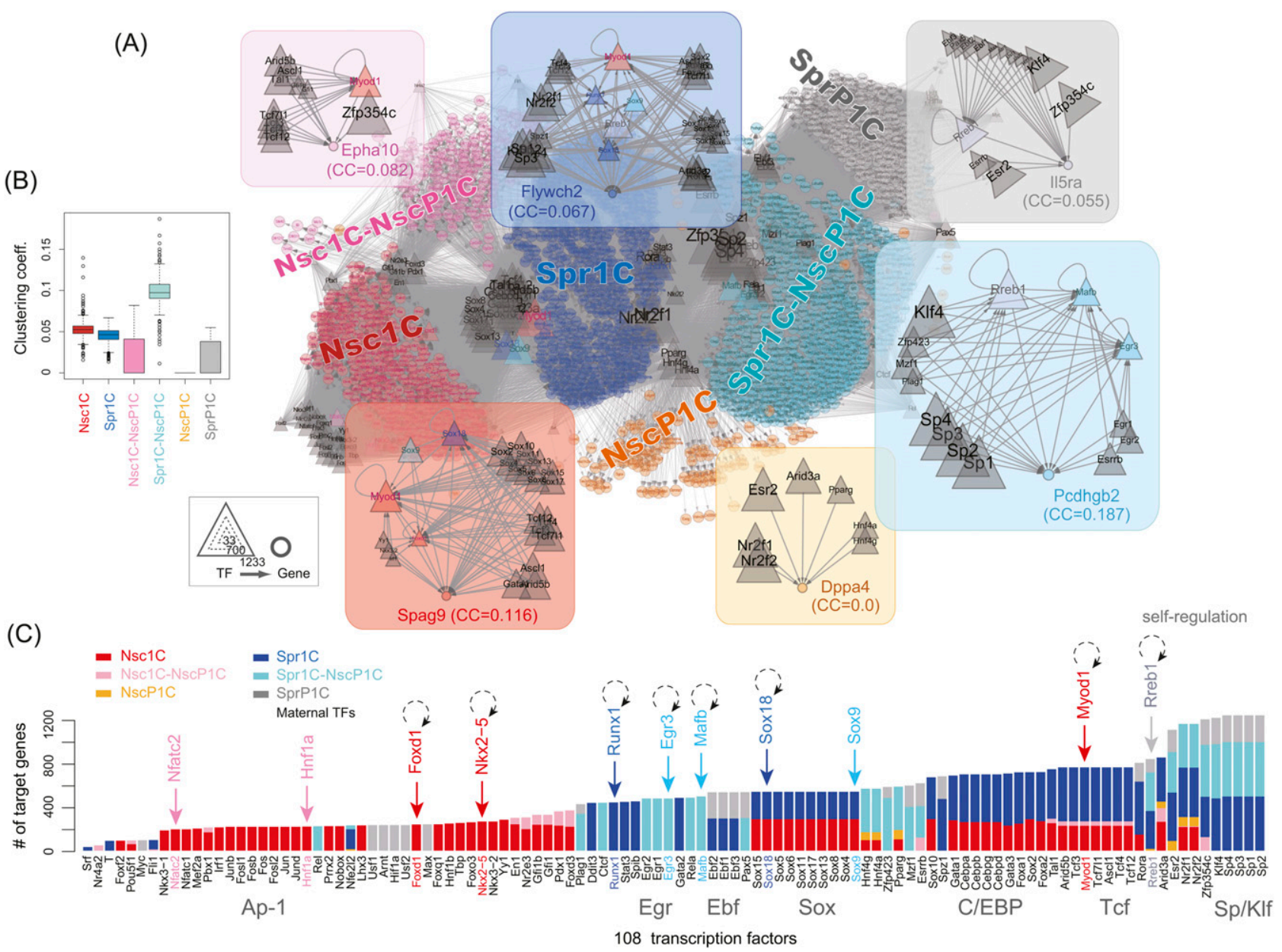

Figure 6. Inference of gene regulatory network. (A) Network including 53,229 directed edges among 1897 nodes (Supplemental Fig. S6 in high resolution). The edge direction is from $108 \mathrm{TFs}$ (triangles) to target genes (circles). Dark-gray triangles represent TFs pooled at oocytes. The inset emphasizes the highest CC of non-TF genes in each category. $(B)$ Overall distribution of CCs. $(C)$ Distribution of targeted genes by 108 TFs. Color corresponds to the gene categories shown in Table 3. (Coeff.) Coefficient. 
lation of the maternal and paternal genomes using inherited genetic and epigenetic molecules and by activating regulatory programs. During fertilization, the oocyte must accomplish a complex series of remarkable tasks, including completion and resumption of the cell cycle, remodeling of parental chromatin, and degradation and transition of maternal mRNAs (Schultz 2002; Wang and Dey 2006). Simultaneously, the sperm must deliver supercompacted paternal genome and other important factors to trigger oocyte activation (Ostermeier et al. 2004; Krawetz 2005; Miller et al. 2010; Erkek et al. 2013).

In this study, we sought to achieve a better understanding of the molecular bases of these dramatic events. To this end, we prepared a very large number of high-quality embryos and exploited high-throughput sequencing technologies. The ultralarge-scale whole-transcriptome data produced in this study showed high reproducibility among replicates, with improved coverage and/or depth. Our extensive transcriptome discovery was further complemented through use of comparative analyses.

We confirmed that a considerable transition in gene expression occurs between 1C and 2C (Supplemental Fig. S1). Interestingly, this expression change was observed for both ncRNAs and mRNAs. The amount of ncRNAs and mRNAs in fertilized embryos was greater than in parthenotes. This implies that the interplay between ncRNAs and mRNAs may be essential for normal development. Indeed, asRNAs detected at oocytes and 1C had a clear impact on the level of translation at 2C. BLAT (Kent 2002) exactly aligned the asRNA segments to coding sequences of 52 proteins identified in a proteomic assay (Wang et al. 2010); 41 of these proteins were reduced in abundance in zygotes compared with MII oocytes (Supplemental Table S6). In addition to polyadenylation controls (Oh et al. 2000; Schultz 2002), asRNAs may uncouple transcription from translation. Our analysis of differential gene expression demonstrated a transcriptionally repressed state during development (Fig. 3A), in agreement with previous reports (Schultz 2002; Bui et al. 2011). Therefore, gene silencing appears to be an essential mechanism in development, in a fashion similar to gene activation (Wang and Dey 2006). In good agreement with our observations, parthenotes, which arrest at the peri-implantation period, showed a defect that led to aberrant gene expression (Fig. 3).

We performed a comprehensive bioinformatics analysis to dissect temporal gene expression changes and their functional associations (Fig. 4). Our results showed programmed expression waves that were consistent with the widely accepted model of early embryo development (Wang and Dey 2006). Additionally, our results revealed new patterns of expression. For example, maternal-tozygotic replacement occurred in distinct subsets of maternal RNAs. Also, one gene subset of the minor ZGA was reactivated at $4 \mathrm{C}$. Remarkably, genes belonging to the minor ZGA pattern were largely associated with a broad range of biological processes that first commenced at 1C (Fig. 4C). Through a detailed investigation of 1C, we identified 834 transcripts for 817 genes that were present in this stage and/or in spermatozoa but not in MII oocytes and parthenotes (Table 3). Approximately
$92 \%$ of these genes exhibited a $>1.5$-fold suppression at 1C following $\alpha$-amanitin treatment (Supplemental Fig. $\mathrm{S} 7 \mathrm{C}, \mathrm{D})$, which supports our interpretation of transcriptional activity by the minor ZGA at $1 \mathrm{C}$. These genes were associated with the processes that the minor ZGA initiates at 1C (Supplemental Table S5) and provided support for the biological significance of signal transduction, such as GPCRs (Williams et al. 1996; Saunders et al. 2002) and MAPK (Sun et al. 1999; Kelkar et al. 2003; Fan and Sun 2004; Kawamura et al. 2005; Liu et al. 2010), which were not enriched in parthenotes. Our data suggest that, immediately after fertilization, the minor ZGA program initiates the transient or continuous activities of important biological processes by incorporating newly synthesized and sperm-borne RNAs as the potential triggers of zygotic genome activation.

To our surprise, the promoters regulating nascent RNAs at 1C contained significant TF-binding sites (Fig. 5). This indicates that specific TFs must be precisely recruited to the promoters. We identified 108 candidate TFs and found that 11 appeared after fertilization (Fig. 6C). In particular, Foxd1, Nkx2-5, Sox18, Myod1, and Runx1 were potential sperm-borne or 1C-synthesized TFs that were not present at oocytes or in parthenotes. In addition, the genes that commenced expression after normal fertilization were mostly downstream targets of maternal nonhistone chromosomal HMG proteins known to contribute to transcriptional initiation at 1C (Beaujean et al. 2000; Schultz 2002). These results suggest that to ensure the appropriate gene expression profile, the first genome activation program at $1 \mathrm{C}$ orchestrates maternal and sperm-primed factors by switching on highly distinctive genetic regulatory codes. Other parentally derived molecules, including ncRNAs and chromatinassociated proteins, are also likely to be involved in this program. If the paternal genome is absent, as in parthenotes, promiscuous gene expression occurs in which 2.3 times as many genes than normal are activated.

Throughout this study, we noted differences and similarities between parthenotes and normal embryos. Genes differentially expressed in parthenotes were associated with significantly distinct processes compared with normal embryos (Fig. 3B-E). However, genes first detected in p1C embryos were involved in many of the biological processes that were also noted for 1C (Supplemental Table S5). Therefore, the RNA profile of parthenotes would seem to suggest that a fertilization event had occurred despite the obvious absence of this event. The underlying basis for this apparent molecular similarity with normally fertilized oocytes remains unclear. Possibly, it may in part be explained by the higher interconnectivity of TFs for regulating genes commonly expressed in sperm and $1 \mathrm{C}$ and p1C (Fig. 6B). In other words, the oocytes can supply all of the TFs necessary for reprogramming via alternative pathways if the paternal genome is absent. In p1C embryos, large quantities of maternally derived molecules may be required in the regulatory network as triggers of ZGA (Fig. 6C), which possibly could result in excessive expression of maternal factors and delayed onset of gene activation. From this perspec- 
tive, the presence of paternal factors is apparently essential for fine-tuning gene expression, which parthenotes cannot achieve. This may support a previous report that appropriate manipulation of genomic imprints leads to the normal development of parthenotes containing only maternal genomes (Kono et al. 2004).

In summary, our ultralarge-scale whole-transcriptome analysis of four early embryo stages provides an important resource for deciphering development after fertilization. Further investigations using the database built in this study will provide new insights into mammalian embryogenesis.

\section{Materials and methods}

\section{Cell collection}

We collected MII oocytes from the oviducts of 8- to 12-wk-old superovulated female $\mathrm{BBF} 1$ mice, which are $\mathrm{F}_{1}$ progeny from a cross between BALB/c females and C57BL/6 males. We collected oocytes from 40 superovulated mice each time. After removing cumulus cells, we manually collected high-quality MII oocytes (20-30 oocytes per mouse). These cells were either used for in vitro fertilization or parthenogenetically activated in M16 medium containing $5 \mathrm{mM} \mathrm{SrCl} 2,5 \mathrm{mM}$ EGTA, and $5 \mu \mathrm{g} / \mathrm{mL}$ cytochalasin B for $30 \mathrm{~min}$. In vitro fertilization was carried out in $50 \mu \mathrm{L}$ of medium containing 10-30 oocytes; parthenogenetic activation was also carried out in $50 \mu \mathrm{L}$ of medium but with 50 oocytes. After insemination or activation, 20 zygotes or activated oocytes were transferred to $20 \mu \mathrm{L}$ of fresh M16 medium. Nocodazole $(0.1 \mu \mathrm{g} / \mathrm{mL})$ was added at 11,31 , or $46 \mathrm{~h}$ after insemination to induce cells to arrest at prometaphase of the first, second, or third mitosis, respectively (Fig. 1A). All cultures were examined every $24 \mathrm{~h}$ and also before the addition of nocodazole to check for embryos showing delayed development; these embryos were removed from the cultures. The first, second, and third mitotic embryos were harvested at 22, 46, and $57 \mathrm{~h}$ after insemination, respectively. All cells were washed in PBS containing 0.5\% PVP and stored in liquid nitrogen until used for RNA preparation.

\section{RNA preparation and sequencing}

Total RNA (3-4 $\mu$ g per stage) was prepared from 10,000 cells at each stage using Isogen II (Nippon Gene). To prepare sequence libraries, we first removed rRNA using a Ribo-Zero Gold kit (Epicentre, an Illumina company), which yielded $\sim 500 \mathrm{ng}$ of RNA per stage. Next, we randomly fragmented the RNAs using RNase III provided in the SOLiD Total RNA-seq kit (product no. 4445374). After isolation with gel electrophoresis, we converted 100- to 200-bp RNA fragments to cDNA libraries in a strandspecific manner. Using the standard protocols from the SOLiD system user manual, the cDNA libraries were clonally amplified onto beads with emulsion PCR. We sequenced the library fragments on a SOLiD 5500xl system analyzer using standard run conditions and generated 50-bp single-ended short reads.

\section{$q R T-P C R$}

Total RNA (500 ng) from each stage was primed using Oligo(dT)20, and cDNA was synthesized using Superscript III (Invitrogen). Two percent of the synthesized cDNA and an appropriate set of primers (Supplemental Table S7) were mixed with Kapa SYBR Fast qPCR Master Mix (Kapa Biosystems) and subjected to qPCR following the manufacturer's instructions. All qPCR reactions were performed on an Applied Biosystems 7500 machine.

\section{Read mapping and RNA quantification}

We conducted TopHat (version 1.4.1)-Cufflinks (version 2.0.2) pipeline (Trapnell et al. 2012) with sequenced reads that had an average base call quality of $>18$. The pipeline was coupled with Bowtie (version 0.12.8) (Langmead et al. 2009). We recursively performed TopHat by truncating the $3^{\prime}$ end of unmapped reads and realigning the reads with more stringent parameters. We empirically set the parameters used sequentially as read length, "initial read mismatches," "segment mismatches," and "segment length": $50,3,2$, and $25 ; 46,3,2$, and $23 ; 42,3,2$, and $21 ; 38$, 2,0 , and 19; and 34, 2, 0, and 17 .

Using uniquely mapped reads, we quantified RNA abundance in units of fragments per kilobase of exon per million mapped reads (FPKM) of Cufflinks. After running Cuffcompare, Cuffdiff was used to assess differential gene expression. All transcripts satisfying several conditions (i.e., successful deconvolution, false discovery rate $[\mathrm{FDR}]<5 \%$, complete match of intron chain if an annotation was given, and FPKM $>0.001$ ) were used for further analysis. The pipeline was run either without (Fig. 1B; Table 1) or with (Figs. 1E, 2A) RefSeq annotations. We prepared the reference genome $(\mathrm{mm} 9)$ and RefSeq annotation from the UCSC Genome Browser (http://genome.ucsc.edu) and downloaded the ncRNA annotation from fRNAdb (Kin et al. 2007).

\section{Transcription inhibition assay}

Collection of oocytes from the oviduct, in vitro fertilization, nocodazole treatment, and harvest were carried out as described above, except that zygotes were transferred to $20 \mu \mathrm{L}$ of M16 medium containing $10 \mu \mathrm{g} / \mathrm{mL} \alpha$-amanitin (Sigma, A2263) $3 \mathrm{~h}$ after insemination. Total RNA was prepared using Isogen II (Nippon Gene) from $\sim 2300$ one-cell embryos arrested at first mitosis. For RNA-seq library construction, we used a TruSeq Standard Total RNA sample preparation kit (Illumina, nos. RS-122-2201 and RS-122-2202) and prepared 600 ng of RNAs. Sequencing was carried out on an Illumina HiSeq 2500 (Illumina) with a 100-bp paired-end protocol. We mapped the paired-end short reads $(111,083,303$; two reads) by the pipeline without recursive mapping and gained $\sim 68$ million uniquely mapped reads $(68,474,975$ forward and $67,809,055$ reverse), $96 \%$ of which were properly paired. Using these uniquely mapped reads from one-cell embryos treated with $\alpha$-amanitin, we conducted Cuffdiff to assess $\alpha$-amanitin-sensitive transcripts (Supplemental Fig. S7).

\section{Preparing the public resource}

We ran the pipeline without recursive mapping for RNA-seq data of the Illumina platform: SRA048261 (oocytes), SRA048260 (2C embryos), and DRA000484 (spermatozoa from C57BL/6N males). Recursive mapping was performed for GSE14605 (oocytes) of the SOLiD platform. We downloaded three array-based mRNA profiles: NIA 22K (Hamatani et al. 2004), Affymetrix MOE430 (Zeng et al. 2004), and Agilent 44K (Pfeiffer et al. 2011). If RefSeq information was not present, probe IDs were converted into UniGene IDs, and then the corresponding RefSeq IDs were retrieved. We downloaded mass spectrometry data from two studies (Wang et al. 2010; Pfeiffer et al. 2011) to screen for gene products. The normalized mass spectral count (Wang et al. 2010) was used to estimate the activity of asRNAs. To define promoter 
regions, we used 20,414 TSSs provided by DBTSS (Database of Transcriptional Start Sites, http://dbtss.hgc.jp).

\section{Bioinformatics analysis}

Enrichment of GO terms was analyzed using DAVID bioinformatics resources with BP ALL terms (Huang et al. 2009). Significant terms (EASE score $<0.01$ ) detected by DAVID were grouped into representative ancestor terms in the data set GO Slim2 by CateGOrizer (Hu et al. 2008). The clustering analysis was conducted using the function hclust of R language; Euclidean distances among FPKMs adjusted by quantile normalization and mean centering were clustered using the hierarchical average linkage method. For gene expression patterning, we cut the hierarchical tree at an empirically defined height at which the maternal degradation and the maternal-to-zygotic transition were clearly captured.

Significant TF-binding sites were detected by scanning core promoter regions spanning -1000 to $+200 \mathrm{bp}$ from TSSs with the nonredundant vertebrate JASPAR CORE, consisting of 130 motifs (Vlieghe et al. 2006). After collecting highly matched JASPAR motifs $(>0.8)$ based on matrix similarity scores (Kel et al. 2003), we tested the significance of motif occurrences in three core promoter ranges $(-200$ to $+50,-500$ to +100 , and -1000 to +200 ). This stepwise expansion of promoter regions prevents the possibility of overlooking the position dependency of TF-binding sites. Approximately $80 \%$ of the motifs that were significant $(P<$ 0.01) in the hypergeometric distribution (Fisher's exact test) were found within -500 to $+100 \mathrm{bp}$. Construction of a regulatory network was performed using TRANSFAC professional (released in January 2013), which provides a list of TFs that are known to bind to JASPAR motifs; after converting JASPAR motifs into matrices in TRANSFAC, we gathered mouse TFs targeting the matrices. We retained TFs if the mRNAs of their coding genes were present in our RNA-seq data. Using these potential regulatory links from TFs to promoters, we analyzed the network statistics using Cytoscape 3.0 (http://www.cytoscape.org).

\section{Data access}

RNA-seq data generated in this study have been deposited in the DDBJ (DNA Data Bank of Japan) Sequence Read Archive (DRA) under accession number DRA001066. We also developed the database DBTMEE (Database of Transcriptome in Mouse Early Embryos, http://dbtmee.hgc.jp).

\section{Acknowledgments}

We thank Dr. Takeo Kishimoto and Dr. Yuki Okada for helpful discussions. Computational resources were provided by the supercomputer system at the Human Genome Center, the Institute of Medical Science, the University of Tokyo. This work was supported by the Research Program of Innovative Cell Biology by Innovative Technology by the Ministry of Education, Culture, Sports, Science, and Technology-Japan. S.-J.P. had full access to all of the data in the study.

\section{References}

Amanai M, Brahmajosyula M, Perry AC. 2006. A restricted role for sperm-borne microRNAs in mammalian fertilization. Biol Reprod 75: 877-884.

Beaujean N, Bouniol-Baly C, Monod C, Kissa K, Jullien D, Aulner N, Amirand C, Debey P, Kas E. 2000. Induction of early transcription in one-cell mouse embryos by microinjection of the nonhistone chromosomal protein HMG-I. Dev Biol 221: 337-354.

Bianchi ME, Beltrame M. 1998. Flexing DNA: HMG-box proteins and their partners. Am J Hum Genet 63: 1573-1577.
Bui HT, Wakayama S, Mizutani E, Park KK, Kim JH, Van Thuan N, Wakayama T. 2011. Essential role of paternal chromatin in the regulation of transcriptional activity during mouse preimplantation development. Reproduction 141: 67-77.

Casanova EA, Shakhova O, Patel SS, Asner IN, Pelczar P, Weber FA, Graf U, Sommer L, Burki K, Cinelli P, et al. 2011. Pramel7 mediates LIF/STAT3-dependent self-renewal in embryonic stem cells. Stem Cells 29: 474-485.

Erkek S, Hisano M, Liang CY, Gill M, Murr R, Dieker J, Schubeler D, Vlag JV, Stadler MB, Peters AH. 2013. Molecular determinants of nucleosome retention at CpG-rich sequences in mouse spermatozoa. Nat Struct Mol Biol 20: 868-875.

Fan HY, Sun QY. 2004. Involvement of mitogen-activated protein kinase cascade during oocyte maturation and fertilization in mammals. Biol Reprod 70: 535-547.

Gaillard C, Strauss F. 2000. High affinity binding of proteins HMG1 and HMG2 to semicatenated DNA loops. BMC Mol Biol 1: 1 .

Gazdag E, Rajkovic A, Torres-Padilla ME, Tora L. 2007. Analysis of TATA-binding protein 2 (TBP2) and TBP expression suggests different roles for the two proteins in regulation of gene expression during oogenesis and early mouse development. Reproduction 134: 51-62.

Gilchrist RB. 2011. Recent insights into oocyte-follicle cell interactions provide opportunities for the development of new approaches to in vitro maturation. Reprod Fertil Dev 23: 23-31.

Hamatani T, Carter MG, Sharov AA, Ko MS. 2004. Dynamics of global gene expression changes during mouse preimplantation development. Dev Cell 6: 117-131.

Hess J, Angel P, Schorpp-Kistner M. 2004. AP-1 subunits: Quarrel and harmony among siblings. J Cell Sci 117: 5965-5973.

Hirata T, Amano T, Nakatake Y, Amano M, Piao Y, Hoang HG, Ko MS. 2012. Zscan4 transiently reactivates early embryonic genes during the generation of induced pluripotent stem cells. Sci Rep 2: 208.

$\mathrm{Hu}$ Z-L, Bao J, Reecy J. 2008. CateGOrizer: A Web-based program to batch analyze gene ontology classification categories. Online J Bioinformatics 9: 108-112.

Huang DW, Sherman BT, Lempicki RA. 2009. Systematic and integrative analysis of large gene lists using DAVID bioinformatics resources. Nat Protoc 4: 44-57.

Kanehisa M, Goto S, Sato Y, Furumichi M, Tanabe M. 2012. KEGG for integration and interpretation of large-scale molecular data sets. Nucleic Acids Res 40: D109-D114.

Kawamura K, Kawamura N, Mulders SM, Sollewijn Gelpke MD, Hsueh AJ. 2005. Ovarian brain-derived neurotrophic factor (BDNF) promotes the development of oocytes into preimplantation embryos. Proc Natl Acad Sci 102: 9206-9211.

Kel AE, Gossling E, Reuter I, Cheremushkin E, Kel-Margoulis OV, Wingender E. 2003. MATCH: A tool for searching transcription factor binding sites in DNA sequences. Nucleic Acids Res 31: 3576-3579.

Kelkar RL, Dharma SI, Nandedkar TD. 2003. Expression of Fas and Fas ligand protein and mRNA in mouse oocytes and embryos. Reproduction 126: 791-799.

Kent WJ. 2002. BLAT-the BLAST-like alignment tool. Genome Res 12: 656-664.

Kin T, Yamada K, Terai G, Okida H, Yoshinari Y, Ono Y, Kojima A, Kimura Y, Komori T, Asai K. 2007. fRNAdb: A platform for mining/annotating functional RNA candidates from noncoding RNA sequences. Nucleic Acids Res 35: D145-D148.

Ko MS, Kitchen JR, Wang X, Threat TA, Hasegawa A, Sun T, Grahovac MJ, Kargul GJ, Lim MK, Cui Y, et al. 2000. Largescale cDNA analysis reveals phased gene expression patterns during preimplantation mouse development. Development 127: $1737-1749$. 
Park et al.

Kobayashi H, Sakurai T, Imai M, Takahashi N, Fukuda A, Yayoi O, Sato S, Nakabayashi K, Hata K, Sotomaru Y, et al. 2012. Contribution of intragenic DNA methylation in mouse gametic DNA methylomes to establish oocyte-specific heritable marks. PLoS Genet 8: e1002440.

Kono T, Obata Y, Wu Q, Niwa K, Ono Y, Yamamoto Y, Park ES, Seo JS, Ogawa H. 2004. Birth of parthenogenetic mice that can develop to adulthood. Nature 428: $860-864$.

Krawetz SA. 2005. Paternal contribution: New insights and future challenges. Nat Rev Genet 6: 633-642.

Langmead B, Trapnell C, Pop M, Salzberg SL. 2009. Ultrafast and memory-efficient alignment of short DNA sequences to the human genome. Genome Biol 10: R25.

Latham KE, Garrels JI, Chang C, Solter D. 1991. Quantitative analysis of protein synthesis in mouse embryos. I. Extensive reprogramming at the one-and two-cell stages. Development 112: 921-932.

Liu N, Enkemann SA, Liang P, Hersmus R, Zanazzi C, Huang J, Wu C, Chen Z, Looijenga LH, Keefe DL, et al. 2010. Genomewide gene expression profiling reveals aberrant MAPK and Wnt signaling pathways associated with early parthenogenesis. I Mol Cell Biol 2: 333-344.

Macfarlan TS, Gifford WD, Driscoll S, Lettieri K, Rowe HM, Bonanomi D, Firth A, Singer O, Trono D, Pfaff SL, et al. 2012. Embryonic stem cell potency fluctuates with endogenous retrovirus activity. Nature 487: 57-63.

Miller D, Brinkworth M, Iles D. 2010. Paternal DNA packaging in spermatozoa: More than the sum of its parts? DNA, histones, protamines and epigenetics. Reproduction 139: 287-301.

Oh B, Hwang S, McLaughlin J, Solter D, Knowles BB. 2000. Timely translation during the mouse oocyte-to-embryo transition. Development 127: 3795-3803.

Ostermeier GC, Miller D, Huntriss JD, Diamond MP, Krawetz SA. 2004. Reproductive biology: Delivering spermatozoan RNA to the oocyte. Nature 429: 154

Pfeiffer MJ, Siatkowski M, Paudel Y, Balbach ST, Baeumer N, Crosetto N, Drexler HC, Fuellen G, Boiani M. 2011. Proteomic analysis of mouse oocytes reveals 28 candidate factors of the 'reprogrammome.'. I Proteome Res 10: 2140 2153.

Piko L, Clegg KB. 1982. Quantitative changes in total RNA, total poly(A), and ribosomes in early mouse embryos. Dev Biol 89: 362-378.

Ramadan WM, Kashir J, Jones C, Coward K. 2012. Oocyte activation and phospholipase $\mathrm{C} \zeta$ (PLC $\zeta$ ): Diagnostic and therapeutic implications for assisted reproductive technology. Cell Commun Signal 10: 12.

Santenard A, Ziegler-Birling C, Koch M, Tora L, Bannister AJ, Torres-Padilla ME. 2010. Heterochromatin formation in the mouse embryo requires critical residues of the histone variant H3.3. Nat Cell Biol 12: 853-862.

Saunders CM, Larman MG, Parrington J, Cox LJ, Royse J, Blayney LM, Swann K, Lai FA. 2002. PLC $\zeta$ : A sperm-specific trigger of $\mathrm{Ca}^{2+}$ oscillations in eggs and embryo development. Development 129: 3533-3544.

Schultz RM. 2002. The molecular foundations of the maternal to zygotic transition in the preimplantation embryo. Hum Reprod Update 8: 323-331.

Smith ZD, Chan MM, Mikkelsen TS, Gu H, Gnirke A, Regev A, Meissner A. 2012. A unique regulatory phase of DNA methylation in the early mammalian embryo. Nature 484: 339-344.

Sturmey RG, Reis A, Leese HJ, McEvoy TG. 2009. Role of fatty acids in energy provision during oocyte maturation and early embryo development. Reprod Domest Anim 44: 50-58.
Sun QY, Lax Y, Rubinstein S, Chen DY, Breitbart H. 1999. Mitogen-activated protein kinase and cell cycle progression during mouse egg activation induced by various stimuli. $Z$ Naturforsch C 54: 285-294.

Svoboda P, Flemr M. 2010. The role of miRNAs and endogenous siRNAs in maternal-to-zygotic reprogramming and the establishment of pluripotency. EMBO Rep 11: 590-597.

Tan MH, Au KF, Yablonovitch AL, Wills AE, Chuang J, Baker JC, Wong $\mathrm{WH}, \mathrm{Li}$ JB. 2013. RNA sequencing reveals a diverse and dynamic repertoire of the Xenopus tropicalis transcriptome over development. Genome Res 23: 201-216.

Tang F, Kaneda M, O'Carroll D, Hajkova P, Barton SC, Sun YA, Lee C, Tarakhovsky A, Lao K, Surani MA, et al. 2007. Maternal microRNAs are essential for mouse zygotic development. Genes Dev 21: 644-648.

Tang F, Barbacioru C, Wang Y, Nordman E, Lee C, Xu N, Wang X, Bodeau J, Tuch BB, Siddiqui A, et al. 2009. mRNA-Seq whole-transcriptome analysis of a single cell. Nat Methods 6: 377-382.

Trapnell C, Roberts A, Goff L, Pertea G, Kim D, Kelley DR, Pimentel H, Salzberg SL, Rinn JL, Pachter L, et al. 2012. Differential gene and transcript expression analysis of RNAseq experiments with TopHat and Cufflinks. Nat Protoc 7: 562-578.

Ufer C, Wang CC. 2011. The roles of glutathione peroxidases during embryo development. Front Mol Neurosci 4: 12.

Vassena R, Boue S, Gonzalez-Roca E, Aran B, Auer H, Veiga A, Izpisua Belmonte JC. 2011. Waves of early transcriptional activation and pluripotency program initiation during human preimplantation development. Development 138: 3699-3709.

Vlieghe D, Sandelin A, De Bleser PJ, Vleminckx K, Wasserman WW, van Roy F, Lenhard B. 2006. A new generation of JASPAR, the open-access repository for transcription factor binding site profiles. Nucleic Acids Res 34: D95-D97.

Wang H, Dey SK. 2006. Roadmap to embryo implantation: Clues from mouse models. Nat Rev Genet 7: 185-199.

Wang PJ, McCarrey JR, Yang F, Page DC. 2001. An abundance of $\mathrm{X}$-linked genes expressed in spermatogonia. Nat Genet 27: 422-426.

Wang QT, Piotrowska K, Ciemerych MA, Milenkovic L, Scott MP, Davis RW, Zernicka-Goetz M. 2004. A genome-wide study of gene activity reveals developmental signaling pathways in the preimplantation mouse embryo. Dev Cell 6: 133-144.

Wang S, Kou Z, Jing Z, Zhang Y, Guo X, Dong M, Wilmut I, Gao S. 2010. Proteome of mouse oocytes at different developmental stages. Proc Natl Acad Sci 107: 17639-17644.

Williams CJ, Schultz RM, Kopf GS. 1996. G protein gene expression during mouse oocyte growth and maturation, and preimplantation embryo development. Mol Reprod Dev 44: 315-323.

Wingender E, Chen X, Hehl R, Karas H, Liebich I, Matys V, Meinhardt T, Pruss M, Reuter I, Schacherer F, et al. 2000. TRANSFAC: An integrated system for gene expression regulation. Nucleic Acids Res 28: 316-319.

Worrad DM, Ram PT, Schultz RM. 1994. Regulation of gene expression in the mouse oocyte and early preimplantation embryo: Developmental changes in Spl and TATA boxbinding protein, TBP. Development 120: 2347-2357.

Zeng F, Baldwin DA, Schultz RM. 2004. Transcript profiling during preimplantation mouse development. Dev Biol 272: 483-496. 


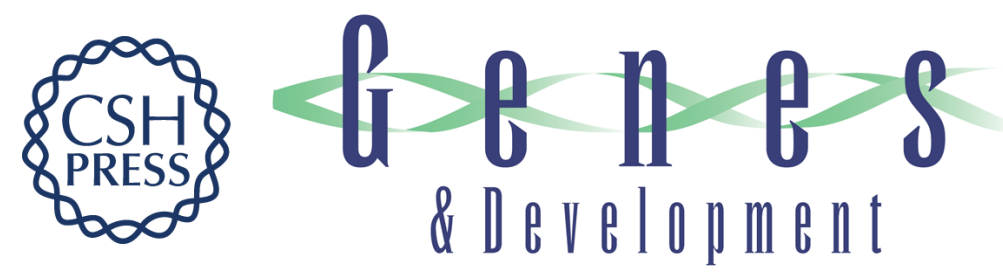

\section{Inferring the choreography of parental genomes during fertilization from ultralarge-scale whole-transcriptome analysis}

Sung-Joon Park, Makiko Komata, Fukashi Inoue, et al.

Genes Dev. 2013, 27:

Access the most recent version at doi:10.1101/gad.227926.113

Supplemental http://genesdev.cshlp.org/content/suppl/2013/12/18/27.24.2736.DC1
Material

References This article cites 57 articles, 17 of which can be accessed free at: http://genesdev.cshlp.org/content/27/24/2736.full.html\#ref-list-1

Creative This article, published in Genes \& Development, is available under a Creative Commons Commons License

Email Alerting Service License (Attribution-NonCommercial 3.0 Unported), as described at http://creativecommons.org/licenses/by-nc/3.0/.

Receive free email alerts when new articles cite this article - sign up in the box at the top right corner of the article or click here.

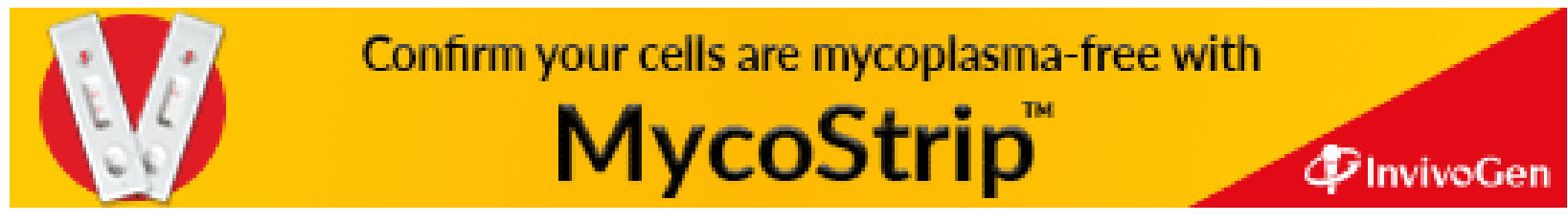

